UDK 821.163.6.09

\author{
Lado Kralj
}

Philosophische Fakultät, Universität Ljubljana

Filozofska fakulteta Univerze v Ljubljani

\title{
Die Darstellung Mitteleuropas in der slowenischen Literatur
}

\author{
Podoba Srednje Evrope v slovenski literaturi
}

\section{ZUSAMMENFASSUNG}

Der Begriff Mitteleuropa hat sich schon seit seiner Entstehung kontinuierlich verändert. Er entstand im deutschen politischen Gebrauch und bezeichnete ursprünglich Deutschland und die Länder östlich davon, auf die sich schon sehr früh die deutschen Interessen richteten. Während des 1 . Weltkriegs entwarf Friedrich Naumann in seinem Buch ein Mitteleuropa als Nachkriegsbund des deutschen und des österreich-ungarischen Reiches, mit besonderem Schwerpunkt auf den Völkern, die weder dem englisch-französischen Bündnis noch dem russischen Zarenreich angehörten. In der Zwischenkriegszeit umfasste der Begriff nicht mehr Deutschland, sondern nur noch die Habsburger Dynastie und die zahlreichen Völker ihres Imperiums. Im Zusammenhang damit stellte sich die Frage, ob Mitteleuropa untrennbar an die Identifikation mit dem habsburgischen Mythos gebunden ist, d. h. mit der Atmosphäre der Sicherheit, Ordnung, eines gemäßigten Konservatismus und liebenswürdiger zwischenmenschlicher Beziehungen, oder wir es auch dann mit Mitteleuropa zu tun haben, wenn wir die Negation dieses Mythos oder eine Polemik damit vorfinden.

Die slowenische Literaturgeschichte stand dem Mitteleuropa-Begriff lange Zeit eher zurückhaltend gegenüber. In früherer Zeit vor allem deshalb, weil sie sehr häufig zu national-konstitutiven Zwecken instrumentalisiert wurde: Ein Volk, das keinen Staat hat, konstituiert sich über seine Literatur. Dieser Standpunkt verschloss jedoch den Zugang zur mitteleuropäischen Idee, die häufig mit der Habsburger Monarchie identifiziert wurde. Gerade von ihr wollten die kleinen Völker sich trennen und eigene
POVZETEK

Zgodovina pojma 'Srednja Evropa' je že od vsega začetka močno spremenljiva. Pojem je nastal v nemški politični rabi in je najprej označeval Nemčijo plus dežele vzhodno od nje, kamor so bili že zelo zgodaj usmerjeni nemški interesi. Med 1. svetovno vojno je Friedrich Naumann v svoji knjigi predvidel Srednjo Evropo kot povojno zvezo Nemškega in Avstro-ogrskega cesarstva, s posebnim ozirom na narode, ki ne pripadajo ne anglo-francoski zvezi ne ruskemu cesarstvu. V obdobju med vojnama pa pojem ni več vključeval Nemčije, temveč samo še habsburško dinastijo in številne narode njenega imperija. V zvezi s tem se je vzpostavila dilema, ali je Srednja Evropa neodtujljivo vezana na identifikacijo s habsburškim mitom, tj. $\mathrm{z}$ atmosfero varnosti, urejenosti, umirjene konservativnosti in ljubeznivih medčloveških odnosov, ali pa imamo opraviti s Srednjo Evropo tudi takrat, kadar zasledimo negacijo tega mita ali polemiko $\mathrm{z}$ njim.

Slovenska literarna zgodovina je bila do pojma 'Srednja Evropa' razmeroma dolgo precej zadržana. V starejšem obdobju predvsem zato, ker je zelo pogosto bila instrumentalizirana $\mathrm{v}$ narodno konstitutivne namene: narod, ki nima države, se konstituira na domači literaturi. To stališče pa je zapiralo dostop do srednjeevropske ideje, saj so jo pogosto identificirali s habsburško monarhijo, in prav od nje so se mali narodi želeli odcepiti in ustanoviti lastne države. Kar zadeva slovensko besedno umetnost, $v$ njej ne najdemo srednjeevropskih značilnosti tako zgodaj kot v glasbeni ali likovni, tj. ne moremo jih locirati že v baroku. Takratna produkcija slovenskih besedil je po obsegu tako skromna, da pač ne more nuditi zadostnega korpusa za preverjanje $\mathrm{v}$ 
Staaten gründen. Im slowenischen Schrifttum finden sich mitteleuropäische Merkmale nicht so früh wie in der Musik und der bildenden Kunst, d. h. wir können sie im Barock noch nicht nachweisen. Die damalige Produktion von slowenischen Texten war ihrem Umfang nach so bescheiden, dass sie keinen ausreichenden Korpus für entsprechende Überprüfungen bieten kann. Dagegen widmete sich die neuere slowenische Literaturgeschichte stärker der Erforschung des literarischen Expressionismus, der sich als eminente literarische Richtung des mitteleuropäischen Raums herausstellt. Im Jahr 1984 begann unter dem Einfluss der Übersetzung von Kunderas Artikel Die Tragödie Mitteleuropas eine Periode der publizistischen Verwendung des Mitteleuropa-Begriffs, die bis zum Fall der Berliner Mauer bzw. bis zur Verselbständigung Sloweniens andauerte. Dabei handelt es sich um eine kulturelle Bewegung von Intellektuellen, überwiegend Schriftstellern, die im Mitteleuropa-Konzept ein Befreiungspotential sahen: Die Identität der mitteleuropäischen Kultur und ihres Geistes sollte den Völkern östlich von Berlin und Wien, die nach dem 2. Weltkrieg von der Sowjetarmee besetzt wurden, zum Widerstand und zur Befreiung verhelfen. Nach dem Fall der Berliner Mauer war die verbindende Funktion des mitteleuropäischen Raums nicht mehr notwendig, da diese bereits von der Europäischen Union ausgeübt wurde. Die Debatte, die bis dahin mit großem emotionalen Einsatz geführt worden war, verlor damit ihre Grundlage und verebbte allmählich. tej smeri. Pač pa se je novejša slovenska literarna zgodovina razživela ob raziskavah literarnega ekspresionizma, ki se izkaže za eminentno literarno smer srednjeevropskega prostora.

L. 1984 se je pod vplivom prevoda Kunderovega članka Tragedija Srednje Evrope začelo obdobje publicistične rabe pojma 'Srednja Evropa', ki je trajalo do padca berlinskega zidu oz. do slovenske osamosvojitve. Gre za kulturno gibanje intelektualcev, večinoma pisateljev, ki so v konceptu Srednje Evrope videli osvobodilni potencial. Identiteta srednjeevropske kulture in duha naj bi narodom vzhodno od Berlina in Dunaja, ki jih je po 2. svetovni vojni okupirala sovjetska armada, pomagala do upora in osvoboditve. Po padcu berlinskega zidu združevalna funkcija srednjeevropskega prostora ni bila več potrebna, saj jo je že opravljala Evropska unija, zato je ta debata, ki se je do takrat razvijala $z$ velikimi emocionalnimi investicijami, polagoma uplahnila.

Der Begriff 'Mitteleuropa' entstand im deutschen politischen Gebrauch und bezeichnete ursprünglich Deutschland und die Länder östlich davon. Wenn die Deutschen sich als Mitteleuropäer bezeichneten, wollten sie sagen, dass sie sich für eine Mittelstellung zwischen dem Westen und dem Osten entschieden hatten und sich sowohl mit dem einen als auch mit dem anderen identifizierten. In der Praxis zeigte sich diese Tendenz erstmals zwischen dem 12. und dem 14. Jahrhundert, als der Osten das Ziel der deutschen Handels- und auch Religionsexpansion wurde. Wie Jacques Le Rider feststellt, gewann in der modernen Zeit der Mitteleuropa-Begriff immer dann an Bedeutung, wenn die deutsche Kultur eine Krise oder eine tiefe Veränderung ihrer geopolitischen Identität erlebte, z. B. nach dem Dreißigjährigen Krieg, nach Napoleon oder nach der Reichsgründung von 1871 (Le Rider 9-10). Eine große Verbreitung erfuhr der Mitteleuropa-Begriff während des 1. Weltkriegs, im Zusammenhang mit dem Militärbündnis zwischen dem Deutschen Reich und ÖsterreichUngarn. Friedrich Naumann entwarf in seinem Buch Mitteleuropa (1915) ein Gebiet, das Deutschland, Österreich-Ungarn und "alle Völker umfassen soll, die weder dem anglo-französischen Westbündnis noch dem Russischen Reich angehören" (Naumann 
9). Das Werk wurde ein Riesenerfolg, in einem Jahr wurden mehr als 100000 Exemplare verkauft, und es brachte den Mitteleuropa-Begriff in den allgemeinen Wortschatz.

In der weiteren Entwicklung des Begriffs kam es vor, dass Deutschland immer weniger eine Schlüsselrolle zugeschrieben wurde, weit mehr dagegen ÖsterreichUngarn, das schließlich bis zum 1. Weltkrieg der reale imperiale Rahmen der meisten mitteleuropäischen Länder gewesen war. Als Brennpunkt der Mitteleuropa-Idee galt Wien, daneben noch Budapest, Prag, Krakau, Zagreb und andere Städte. Mitteleuropa war also nach einigen Interpretationen schlicht und einfach identisch mit der österreich-ungarischen Monarchie. Und als die mitteleuropäischen Völker nach Ende des 1. Weltkriegs ihre Eigenstaatlichkeit erlangten, dürfte diese Gleichsetzung zumindest einige der Befreiten aus dem 'Völkergefängnis' von einer MitteleuropaIdee abgeschreckt haben. Nach dem 2. Weltkrieg verschwand Mitteleuropa schließlich aus dem politischen Lexikon, denn der Eiserne Vorhang hatte Europa in West und Ost geteilt, und der Osten hatte sich auch Mitteleuropa genommen, mit Ausnahme Österreichs, das an den Westen fiel.

Es ist also schwer, Mitteleuropa eindeutig zu definieren, da der Begriff veränderbar, dehnbar und manchmal auch widersprüchlich ist. Das zeigte sich im Juni 1989, einige Monate vor dem Fall der Berliner Mauer, auf einem Symposium in Budapest zum Thema Mitteleuropa. Das Einleitungsreferat hielt Czeslaw Milosz, der Mitteleuropa definierte als "alle Staaten, einschließlich der baltischen, die im August 1939 der reale oder hypothetische Gegenstand des Handels zwischen der Sowjetunion und Deutschland waren" (Budapest Roundtable 18). Dem widersprach scharf der Österreicher Carl Artmann: Österreich sei ein integraler Bestandteil Mitteleuropas und Milosz habe nicht das Recht, Mitteleuropa nur auf die Länder des europäischen Ostens zu beschränken, die von der Sowjetunion besetzt wurden. Österreich habe sich relativ schnell aus der Besatzung befreien können, doch dürfe man es dafür nicht bestrafen (22). Die dritte Interpretation präsentierte Claudio Magris. Mitteleuropa dürfe man, so Magris, nicht mit dem deutschen historisch-politischen Begriff Mitteleuropa gleichstellen. Dieser bezeichne "den Konflikt der deutschen Kultur mit anderen Kulturen in dieser Region" und er setze "die deutsche bzw. deutsch-ungarische Vorherrschaft voraus" (29). Es ist bekannt, dass Magris, anstatt mit 'Mitteleuropa' mit den Begriffen 'der habsburgische Mythos', 'die habsburgische Kultur' und der 'Donauraum' operiert. Drei stark engagierte Schriftsteller aus dieser Region haben also drei sehr unterschiedliche Konzepte: Mitteleuropa ohne Wien - Mitteleuropa mit Wien und das habsburgische Wien mit dem Donauraum, was aber nicht Mitteleuropa ist.

$\mathrm{Zu}$ den slowenischen Schriftstellern kommen wir noch. Doch zuerst muss man darauf hinweisen, dass sich mitteleuropäische Merkmale im slowenischen Schrifttum nicht so früh finden wie in der Musik und der bildenden Kunst, wir sie also nicht schon im Barock feststellen können. Die damalige Produktion slowenischer Texte ist ihrem Umfang nach so bescheiden - Janez Svetokriški (Ioannes Baptista a Santa Cruce), Rogerij Ljubljanski (Rogerius Labacensis), Processio Locopolitana - dass sie keinen ausreichenden Korpus für derartige Untersuchungen bieten kann. Was aber die slowenische Literaturgeschichte anbelangt, hat sich diese auch bei der Erforschung 
späterer Perioden nur ungern und selten mit der Frage der europäischen Identität befasst, und so fand sie wichtigere Indizien dafür erst in der slowenischen Literatur Ende des 19. Jahrhunderts, völlig zuverlässige dagegen in der Zwischenkriegszeit. Woher diese Zurückhaltung der slowenischen Literaturgeschichte? Früher lag der Grund wahrscheinlich darin, dass bei den Slowenen, ähnlich wie bei anderen kleinen Völkern des mitteleuropäischen Raums, die Literaturgeschichte ein besonderes Problem war; sehr häufig wurde sie nämlich zu national-konstitutiven Zwecken instrumentalisiert. Die slowenische Literaturgeschichte hatte sich bis vor kurzem fast gänzlich dieser Pflicht verschrieben, wenn auch nicht immer ohne selbstkritische Reflexionen. So schreibt z. B. Ivo Grafenauer, der wahrscheinlich erste moderne slowenische Literaturhistoriker, schon 1909 im Vorwort zu seiner Geschichte der modernen slowenischen Literatur. "Die politischen Ziele des erwachenden Volkes treten so stark in den Vordergrund, dass die literarische Kunst ihre Magd wird. Die Literatur, die Poesie hat eine Bedeutung nur als patriotisches Werk" (Grafenauer 2). Oder, wie viel später Cornis-Pope und Neubauer feststellen: "Die Literatur in der Volkssprache war oft ein Präludium zur Staatsbildung oder gar die Voraussetzung dafür" (CornisPope/Neubauer 12). Bei diesem Stand der Dinge war die Idee eines Mitteleuropa natürlich störend, da sie für viele identisch war mit der österreichisch-ungarischen Monarchie, und von ebendieser Monarchie musste man sich, so schien es, so bald wie möglich emanzipieren und völlig unabhängig eine ganze Reihe kleiner, neuer Staatengebilde erschaffen.

Wenn wir uns umsehen, stellen wir die etwas weniger bekannte Tatsache fest, dass die Literatur nicht nur bei den kleinen Völkern zu national-konstitutiven Zwecken instrumentalisiert wurde. Tatsächlich begann dieser Prozess, charakteristisch für das 19. Jahrhundert, in jenen Gesellschaften, die Probleme mit ihrer Identität hatten, und das waren Deutschland, Italien und einige skandinavische Länder. Deutschland spielte hier eine paradoxe Rolle: Die grundlegenden Ideen über die nationale Literatur stammten von Herder und den deutschen Romantikern und wurden dann für das nationale Erwachen der kleineren Völker in Ost- und Südosteuropa verwendet. "Deutschland exportierte sein Identitätsproblem nach Osten und verschlechterte es dadurch noch" (Cornis-Pope/Neubauer 12).

So die ältere Literaturgeschichte. Die neuere Literaturgeschichte in Slowenien steht dem Konzept Mitteleuropa zurückhaltend gegenüber aus Gründen, die Janko Kos genauer formuliert. Kos stellt folgende Fragen: Kann das Phänomen der mitteleuropäischen Literatur mit literaturhistorischer, d. h. wissenschaftlicher Strenge erforscht werden, oder geht es lediglich um eine aktualistische und publizistische Tendenz? Diese wurde geboren, um Janko Kos zu ergänzen, aus dem brennenden Wunsch einiger Intellektueller in den 80er Jahren, dass die zahlreichen kleinen Nationen, die von der Sowjetunion nach dem 2. Weltkrieg ihrer europäischen Identität beraubt wurden, durch einen gemeinsamen Auftritt im Namen einer historischen (mitteleuropäischen) Idee die Fesseln dieser sowjetischen Besatzung sprengen. Die Idee einer mitteleuropäischen Kultur bzw. eines mitteleuropäischen Geistes sei, so Kos, von Anfang an von einer inneren Widersprüchlichkeit belastet, von einer konfliktträchtigen Zerrissenheit zwischen dem habsburgischen Mythos auf der einen und 
den antihabsburgischen Befreiungstendenzen der Völker des Donauraums auf der anderen Seite. Die Einheit der mitteleuropäischen Kultur kann nur vom habsburgischen Mythos ausgehen, einen anderen Ursprung hat sie nicht. Kos definiert den habsburgischen Mythos als ein besonderes Verhältnis zur Welt, das sich gegen den nationalen, politischen, moralischen und sozialen Radikalismus wendet, ein Verhältnis, das

einem gemäßigten Konservatismus erwächst, einem rationalen Savoir-vivre und dem Willen zur Erhaltung des Status quo in einer Welt der Vernunft, eines Gleichgewichts der Kräfte, der liebenswürdigen Zwischenmenschlichkeit, vor allem aber der Sicherheit, deren Garant mehrere Jahrhunderte die Habsburger Monarchie mit ihrer rationalen Bürokratie, den Reformbestrebungen und ihrer zivilrechtlichen Ordnung war (Kos 51).

Und dieser Mythos, dieses besondere Weltgefühl sei charakteristisch nur für die österreichische, nicht aber auch für andere Literaturen des Donauraums: "Möge dieser habsburgische Mythos noch so wichtig sein für den 'Geist' der neueren österreichischen Literatur von Grillparzer bis zu den nostalgischen Gefühlen bei Roth oder Musil, muss man natürlich darauf hinweisen, dass er keinesfalls typisch ist für die Literatur in anderen mitteleuropäischen Ländern."Vielleicht ist Prof. Kos bei seinem Schluss etwas zu rigoros, doch ist seine Analyse deshalb sehr wichtig, weil er in die Mitteleuropa-Debatte schon früh, im Jahre 1991 'Magris' Begriff des 'habsburgischen Mythos' eingeführt und ihn als unverzichtbaren Referenzpunkt aufgestellt hat. Claudio Magris prägte den Begriff 1963 in seinem Buch Il mito asburgico nella letteratura austriaca moderna, doch weckte das Werk erst im Jahr 2000 größere Aufmerksamkeit, als es in Wien in deutscher Übersetzung erschien.

Man muss aber mindestens ein Segment anführen, wo die neuere slowenische Literaturgeschichte das Mitteleuropa-Konzept dennoch als relevante Grundlage der wissenschaftlichen Forschung ansieht: bei Studien über den literarischen Expressionismus. Dieser entstand in Berlin und weitete sich dann über Wien fast ausschließlich auf die Literaturen der mitteleuropäischen Völker aus. Er tauchte auf in der slowenischen, kroatischen, ungarischen, tschechischen, slowakischen, polnischen, rumänischen, teilweise auch in der bulgarischen und serbischen Literatur (vgl. Kralj 71-117). Wenn wir also den literarischen Expressionismus als internationale Erscheinung ansehen, ist das ausschließlich eine Domäne Mitteleuropas - die französische und die angelsächsische Literatur blieben ihm verschlossen. Manchmal trifft man in literaturhistorischen Abhandlungen anstatt der Bezeichnung 'Mitteleuropa' auch auf poetische Synonyme: 'die Landschaften östlich von Triest' (S. Wollmann), 'europäisches Zwischenfeld' oder 'litteratura Danubiana' (Z. Konstantinović, und auch andere). Die geographische Begrenzung des Expressionismus auf Mitteleuropa ist umso ungewöhnlicher, wenn wir die Verbreitung anderer großer literarischer Richtungen berücksichtigen, z. B. der Romantik, des Naturalismus oder des Symbolismus, die als neue, schöpferische Initiativen immer von ganz Europa und auch von der ganzen Welt angenommen wurden. Sofort muss man auch den etwaigen Einwand zurückweisen, dass diese geographische Begrenzung ausschließlich die Folge des deutschen kulturellen Einflusses auf die erwähnten Literaturen und Völker ist, da uns schon ein flüchtiger Blick zeigt, dass zahlreiche Werke der expressionistischen Lite- 
ratur ausdrücklich mit Österreich-Ungarn polemisieren. Sie erheben den Vorwurf, es sei ein 'Völkergefängnis' und prangern das Vorgehen der deutschen und der österreichischen Armee im 1. Weltkrieg an. Kurzum: Mitteleuropa und der literarische Expressionismus sind völlig komplementär. Einerseits zeigt sich das Wesen Mitteleuropas am überzeugendsten im Expressionismus, andererseits hat sich der Expressionismus vor allem in Mitteleuropa ausgebreitet, woanders dagegen fast gar nicht.

Die Wechselwirkungen zwischen den einzelnen nationalen Literaturen im mitteleuropäischen Raum waren so stark und charakteristisch, meint Zoran Konstantinović, dass man von einem 'interethnischen Strukturmodell der Literatur' sprechen müsse. Hier verflechten sich geographische, soziologische und literaturhistorische Gesichtspunkte. Konstantinović spricht von der Einheit der politischen und sozialen Struktur innerhalb des mitteleuropäischen Raums (man könnte hinzufügen, dass es sich eigentlich um den habsburgischen 'Ordo' handelt, die habsburgische Gesellschaftsordnung) und das diese "die Gemeinschaft in der Entwicklung und in den geistigen Manifestationen" zur Folge hat. Ein charakteristischer gemeinsamer Zug ist z. B., dass die expressionistische Literatur in Mitteleuropa mehrheitlich eine Überdosierung des avantgardistischen Formalradikalismus vermeidet und sich nicht völlig von der literarischen Tradition entreißen kann, d. h. vom Symbolismus, oder sie greift manchmal noch weiter zurück, bis zu folkloristischen Elementen. Diesen Feststellungen von Konstantinović könnte man z. B. die einheitliche Periodisierung des Expressionismus in der mitteleuropäischen Literatur hinzufügen: Diese begann sozusagen ohne Ausnahme mit dem Jahr 1918, d. h. mit dem Ende des 1. Weltkriegs, der deutsche Expressionismus begann dagegen schon 1910. Das ist noch immer ein achtjähriger Rückstand, doch erschien er den Literaturhistorikern verschwindend gering und im Vergleich zu der langsamen Rezeption früherer literarischer Richtungen empfanden sie das als großen Fortschritt. Fran Petrè stellte z. B. 1954 über den slowenischen Expressionismus zufrieden fest, dies sei "die erste literarische Strömung in der slowenischen Entwicklung, die nicht mit Verspätung" eingesetzt habe (Petrè 643).

In neuerer Zeit arbeiten an der Erforschung der Literaturen Mitteleuropas eifrig die Mitarbeiter einer umfassenden internationalen Studie. Sie kommen aus aller Welt - es sind mehr als 120 - und sie handeln nach dem Motto "Rethinking Literary History". Sie versuchen also, neue methodologische Modelle anzubieten und damit auf die heftige Kritik zu antworten, die die klassische Geschichtsschreibung und mit ihr auch die Literaturgeschichte erlebt. Die Kritik richtet sich vor allem gegen die chronologische, kontinuierliche Darbietung von historischem Material, mit dem Vorwurf, es sei manipulativ, ideologisch, daneben mehr fabulierend als wissenschaftlich. Die Mitarbeiter gehen davon aus, dass sich die vorgeworfenen Fehler vermeiden lassen, wenn man die Logik des nationalen Interesses überwindet, $d$. h. die Logik einer Literaturgeschichte, die die Schaffung einer Nation unterstützt. Zu diesem Zweck muss ein größeres und vertikal übergeordnetes Untersuchungskorpus ausgewählt werden. Ein solches Korpus haben die Mitarbeiter bereits bearbeitet und eine Literaturgeschichte in drei Teilen verfasst mit dem Titel Oxford Comparative History of Latin American Literary Cultures. Neben einer anderen Korpusauswahl liegt eine der methodologischen Innovationen auch darin, dass in möglichst hohem Maße die übliche Chrono- 
logie durch ein Netz miteinander verbundener 'Knoten', d. h. kulturologischer Querschnitte, ersetzt wird. Das Korpus im ersten Projekt war also das gesamte Lateinamerika; das zweite Projekt, das noch auf den Druck wartet und ebenso in 3 Teilen erscheinen wird, trägt den Titel History of the Literary Cultures in East-Central Europe: Junctures and Disjunctures in the 19th and 20th Centuries. Der Reichtum in der Vielfalt, die große Zahl der behandelten Literaturen (oder, im zweiten Projekt, sogar Sprachen), die Dynamik der Interaktionen zwischen ihnen - all das soll eine andere, lebendigere, obwohl natürlich weniger präzise, umfassende und systematische Literaturgeschichte ermöglichen, denn der Leser bekommt auf aleatorische Art und Weise über ein gewünschtes Segment nur so viel Material, wie es das Netz der 'Knoten' im jeweiligen Fall ermöglicht.

Versuchen wir mit Hilfe dieses Netzes die Frage zu beantworten, ob die slowenische Literatur zu Mitteleuropa gehört. Dazu verwenden wir einen der Knoten - Städte als Schauplätze der hybriden Identität und polykulturellen Produktion. Wurden die slowenischen Literaten in einer solchen Stadt geformt? Die Antwort ist wahrscheinlich 'ja', denn Wien entspricht sehr gut dieser Beschreibung. Die Identität Wiens war auf verschiedene Weisen hybrid und polykulturell, hier floss das gesamte österreichisch-ungarische Imperium zusammen, und Proletarier mit tschechischen Namen kennen wir gut aus den Werken von Ivan Cankar. Natürlich schöpften die slowenischen Literaten schon seit Prešeren aus der geistigen Atmosphäre Wiens, denn sie studierten an der Wiener Universität (die slowenischen Humanisten noch früher, im 15. Jahrhundert). Das Verhältnis zu Wien war komplex, zum Teil antagonistisch, zum Teil symbiotisch, denn Wien war der Mittler nicht nur der deutschen, sondern jeder fremden Kultur und Literatur, jedes internationalen Einflusses, z. B. von mindestens drei literarischen Richtungen: des Naturalismus, Symbolismus und Expressionismus. Das war ein Prozess, den sehr gut der Titel einer angesehenen katholischen Zeitschrift der Zwischenkriegszeit ausdrückt - Dom in svet (Heim und Welt). Die slowenische Literatur musste das schwierige Gleichgewicht halten zwischen Heim und Welt, zwischen dem Prinzip der Traditionserhaltung und der Öffnung für Neues, Fremdes, Internationales. Und die 'Welt' war für die Slowenen sehr lange, bis zum 2. Weltkrieg, Wien; hier lebte einst eine recht zahlreiche Kolonie slowenischer Studenten. Josip Stritar beispielsweise trennte sich von Wien (oder seiner Umgebung) überhaupt nicht mehr, bis er zum Sterben in die Heimat zurückkehrte.

Die Repräsentation Wiens als moderne Großstadt, gleichzeitig auch als Ort des geistigen Leidens, ist bei Prešeren und Stritar noch abwesend, besonders scharf ist sie bei Ivan Cankar und danach noch bei Slavko Grum oder Stanko Majcen. Beim Gegensatz zwischen 'Heim' und 'Welt' neigt sich die 'Welt' zur Negativität. Erinnern wir uns nur an das Wiener Arbeitermilieu in der Vorstadt Ottakring, dieser mitteleuropäischen urbanen Hölle, in der unglückliche Seelen aus allen Ecken der Monarchie dahinsiechten, wie wir aus Cankars Erzählungen Das Haus der Barmherzigkeit und Nina ersehen können. Diese Atmosphäre wird völlig verschärft und grotesk deformiert in Grums Drama Das Ereignis in der Stadt Goga oder auch in seinen Kurzgeschichten, die als Material dazu anzusehen sind. Die fiktive Stadt Goga, die auf den Fundamenten von Cankars Spuk im Florianital entstand, ist der umfassends- 
te slowenische Beitrag zur literarischen Repräsentation Mitteleuropas, obwohl das Bild wegen seiner grotesken Verzerrung nicht sehr angenehm ist. Das Grundmotiv ist das Bedürfnis nach Gemütlichkeit, das wir auch als Bedürfnis nach Sicherheit und Schutz interpretieren können. Es geht natürlich um eine Variante des 'habsburgischen Mythos', bzw. der Ordnung, um Nostalgie nach dem gesicherten und konservativen Status quo, nach einer Welt, die sich nicht verändert und in der die Menschen miteinander liebenswürdig und etwas jovial verkehren. Deshalb ist die Stadt Goga statisch, verschlafen, mehr einem provinziellen Marktflecken ähnlich als einer Großstadt. Und trotzdem, trotz dieser Schutzmaßnahmen geschehen in Goga schreckliche Dinge, die Ermordung eines Kindes außerhalb der Stadt, in der Stadt wiederholte Vergewaltigungen und ein weiterer Mord. Deshalb arbeitet die Gesellschaftsordnung als ununterbrochene Überwachungsmaschine, jeder überwacht jeden, was große Beklommenheit auslöst. Dieses Syndrom nennt Freud, ein großer Kenner der mitteleuropäischen Verhältnisse, das 'Unbehagen in der Kultur'. Die Gemütlichkeit, die sich nach außen zeigt, verdeckt oder verdrängt immer voluntaristisch eine existentielle Beklommenheit, die früher oder später manifest herausbricht, am pragmatischsten bei Franz Kafka. In Majcens expressionistischer Literatur, vor allem in seinen Dramen, breiten sich die Kontrolle und die Beklommenheit aus dem 'Heim' auf die 'Welt' aus, auf den mitteleuropäischen, multinationalen Raum: Vor Grauen wahnsinnig gewordene Ukrainer warten im Gefängnis auf ihre Erschießung durch die österreichische Soldateska (der Einakter Apokalypse), die serbische Zivilbevölkerung wird arrogant malträtiert von der österreichischen Besatzungsarmee, die multinational ist, denn unter den Offizieren sind auch ein Slowene und ein Jude (Kasija). Eine solche Vogelperspektive auf den mitteleuropäischen Raum wird natürlich ermöglicht durch den 1. Weltkrieg, dieses traumatische und wichtige expressionistische Thema, so auch in France Bevks Einakter In der Tiefe oder im Kriegstagebuch von Andrej Čebokli.

Diesen schnellen Überblick über die mitteleuropäischen Merkmale der slowenischen Literatur muss man wahrscheinlich mit Grum auch beenden, genauer: beenden muss man ihn Ende der 30er Jahre, d. h. am Anfang des 2. Weltkriegs. Die Zwischenkriegszeit war nämlich dem habsburgischen Mythos verbunden, die Zeit nach dem 2. Weltkrieg dagegen überhaupt nicht mehr. Deshalb bekommt das Mitteleuropa-Konzept, das nach langer Zeit wieder auftaucht, neue, andersartige Züge und Ziele.

Der angesehene tschechische Schriftsteller Milan Kundera veröffentlichte im November 1983, sechs Jahre vor dem Fall der Berliner Mauer, in der französischen Zeitschrift Le débat den Essay Die Tragödie Mitteleuropas, der im Jahr darauf im New York Review of Books als Nachdruck erschien (26. April 1984), um sich dann in Übersetzungen explosionsartig über die gesamte Weltpresse auszubreiten. Schriftsteller und Intellektuelle, die hinter dem Eisernen Vorhang lebten oder von dort emigriert waren, erkannten sofort, dass Kundera etwas angesprochen hatte, "was uns schon lange nicht nur auf den Herzen, sondern auch auf der Zunge lag", so Drago Jančar (Jančar 88): Er löste das Befreiungspotential Mitteleuropas aus. Die Russen hatten 
Mitteleuropa besetzt, hatten dieses weite Territorium zu einem farblosen Lager gemacht, zum Ostblock, und den mitteleuropäischen Geist der Ausrottung und dem Vergessen preisgegeben. Der Westen ließ dieses Sterben ruhig zu, eigentlich bemerkte er es gar nicht mehr. Und nun hatte Kundera den Geist Mitteleuropas wiedererweckt, er berief sich auf die einstige Einheit und schuf aus einer Reihe einzelner, untereinander sogar zerstrittener Völker die Hoffnung auf etwas, was einer Front ähnelte, etwas, was der Westen vielleicht unterstützen würde.

Die Prämisse von Kunderas Konzept ist, dass Europa schon immer in zwei Hälften geteilt war, die sich getrennt entwickelten: "Das eine war an das antike Rom und die katholische Kirche gebunden, das andere in Byzanz und der orthodoxen Kirche verankert. Nach 1945 verschob sich die Grenze zwischen den beiden Europas mehrere hundert Kilometer nach dem Westen, und verschiedene Völker, die sich immer westlich gefühlt hatten, wachten auf und mußten feststellen, daß sie nun im Osten waren" (Kundera 133). Mitteleuropa, das sind also vor allem jene Völker und Gebiete östlich von Berlin und Wien und westlich von Moskau, die nach dem Ende des 2. Weltkriegs von der russischen Armee besetzt wurden - und ein solches Verständnis von Mitteleuropa hat das Bewusstsein der Schriftsteller und Intellektuellen aus diesem Gebiet entscheidend mitgeprägt, denn noch kurz vor dem Fall der Berliner Mauer verteidigte es Czeslaw Milosz bei dem erwähnten Symposium in Budapest, obwohl in präzisierter Form. Das heißt aber, dass der Begriff in seinem modernen Gebrauch nach 1983 wieder mit einem sehr bestimmten Profil ins Leben gerufen wurde, das in der Geschichte bis dahin unbekannt war, und das dieses Profil vor allem politischer und ideologischer Natur war. Der Begriff konnte sich natürlich aufgrund seiner großen Beliebtheit schnell entwickeln und ausweiten und er übernahm mit der Zeit auch einige historiographische und kulturologische Merkmale aus seinen früheren Bedeutungen, doch der Schwerpunkt des neu errichteten Begriffs blieb dennoch politisch. Mitteleuropa wurde schlicht und einfach als Befreiungsmotto im Kampf gegen den Kommunismus verstanden. Der Kommunismus aber wurde gleichgesetzt mit Russland, mit der russischen Weltanschauung:

Die Welt der Russen fasziniert uns und zieht uns an - vorausgesetzt, wir sind in Distanz zu ihr; in dem Moment, in dem sie sich um uns schließt, enthüllt sie ihre schreckliche Fremdheit. Ich weiß nicht, ob sie schlechter ist als unsere, aber ich weiß, daß sie anders ist: Rußland kennt andere (größere) Dimensionen des Unglücks, hat eine andere Vorstellung von Raum (ein Raum, so riesig, daß ganze Völker von ihm geschluckt werden), einen anderen Zeitbegriff (langsam und geduldig), eine andere Art zu lachen, zu leben und zu sterben. Deshalb empfinden die Länder Mitteleuropas ihr Schicksal nach 1945 nicht nur als eine politische Katastrophe: es ist auch ein Angriff auf ihre Zivilisation. Der tiefere Sinn ihres Widerstandes ist der Kampf um die Erhaltung ihrer Identität - oder anders ausgedruckt: um die Erhaltung ihrer 'Westlichkeit' (Kundera 136-137).

Mit diesem Mitteleuropa-Konzept, das als Motto des Widerstands gegen den Kommunismus bzw. gegen Russland formuliert wird, wendet sich Kundera an die Intellektuellen aus der literarischen Sphäre und fordert sie auf, sich politisch zu engagieren ein Modell, das davor in den 60er Jahren von J. P. Sartre erstellt wurde, jedoch in einem anderen, genau umgekehrten politischen Kontext. Das Modell setzt voraus, dass die 
MUZIKOLOŠKI ZBORNIK • MUSICOLOGICAL ANNUAL XL

Literatur besondere Macht hat; deshalb kann ein engagierter Schriftsteller ein erfolgreicher Kämpfer für humanistische Ziele sein. Er engagiert sich, indem er nicht mehr nur auf dem Gebiet der Ästhetik schreibt, sondern auch auf dem Gebiet der politischen Aktion. Dabei ging Kundera, ob er sich dessen bewusst war oder nicht, von einer älteren Vorstellung des Begriffs Mitteleuropa aus, von der wir schon gesprochen haben: dass die Literatur in der Nationalsprache ein Präludium zur Staatsformung oder sogar die Voraussetzung dafür ist. Die gesamte Vorstellung Kunderas von Mitteleuropa stützt sich also auf das Axiom über die besondere Macht der Literatur bzw. Kultur:

Die Identität eines Volkes oder einer Zivilisation wird reflektiert von und ist konzentriert in dem, was von Geist und Seele geschaffen wurde - in dem, was man gemeinhin unter 'Kultur' versteht. Wenn diese Identität ausgelöscht zu werden droht, wird das kulturelle Leben entsprechend intensiver und wichtiger, bis die Kultur selbst zum Lebenswert wird, um den sich alle Menschen scharren. Deshalb spielen in jeder Revolution in Mitteleuropa das gemeinsame kulturelle Erbe und die zeitgenössischen kreativen Anstrengungen eine so große und entscheidende Rolle - größer und entscheidender als je in einer anderen europäischen Massenrevolution (Kundera 134).

Wenn er schon den Begriff Mitteleuropa wiedererweckte, musste er auch zum habsburgischen Mythos Stellung beziehen, dessen war sich Kundera bewusst. Das tat er wie folgt: Weil Mitteleuropa sich aus einer so großen kulturellen Vielfalt zusammensetzt, ist es sein Idealziel, ein Europa im Kleinen zu werden, ein verkleinertes Modell von Europa. Und diese Möglichkeit bot sehr wohl schon, bot unersetzlich das österreich-ungarische Imperium, das seine Bewohner in ihrer Unwissenheit gesprengt hatten. "Das österreichische Kaiserreich hatte die große Chance, Mitteleuropa zu einem starken geeinigten Staat zu machen. Aber die Österreicher waren leider selbst hin- und hergerissen zwischen einem arroganten pangermanischen Nationalismus und ihrer eigenen mitteleuropäischen Mission. Es gelang ihnen nicht, eine Föderation gleichberechtigter Nationen zu bilden, und ihr Scheitern war das Unglück für ganz Europa. Die anderen Nationen Mitteleuropas sprengten in ihrer Unzufriedenheit ihr Reich 1918 auseinander, ohne zu begreifen, dass es trotz seiner Unzulänglichkeiten unersetzlich war" (Kundera 137).

Kunderas Artikel löste eine Flut des Beifalls, der Unterstützung und weiterer Beiträge aus, oder, wie Drago Jančar es formulierte: "Das mitteleuropäische Thema erblühte auf den Seiten der Kulturzeitungen" (Jančar 93), natürlich vor allem in den Ländern mit kommunistischen Regimes. Einer der angesehendsten Unterstützer von Kunderas Konzept. wurde der ungarische Schriftsteller György Konrád, der in den 90er Jahren auch Präsident des Internationalen P.E.N. und Präsident der Berlin-Brandenburgischen Akademie der Künste war. In seinem Artikel Der Traum von Mitteleuropa stellte Konrád einige wichtige neue Aspekte vor. Einerseits trug er einige Vorbehalte zusammen: Es stimmt, die Idee eines Mitteleuropa dürfte konservativ sein, oder es ist eine Utopie, oder es sind vielleicht nur Träume, wie schon der Titel impliziert, doch auf der anderen Seite hat diese konservative, utopische, träumerische Idee einige unumstrittene Qualitäten. Sie ist visionär und vermutlich haben die Visionen eine Chance, verwirklicht zu werden. Und das Ziel der Visionen? Eine Konföderation. "Die mitteleuropäische Konföderation durch unsere kulturellen und per- 
MUZIKOLOŠKI ZBORNIK • MUSICOLOGICAL ANNUAL XL

sönlichen Beziehungen vorwegzunehmen - ein kühner Versuch!" In einer solchen Konföderation gälte das mitteleuropäische Prinzip der 'blühenden Vielfalt der Bestandteile, des Selbstbewusstseins der Diversität'. Das ist natürlich nur eine Vision und Mitteleuropa bleibt für Konrád noch immer ein komischer Klub, aber dennoch: "Ein komischer Klub, zu dem es sich lohnt, dazuzugehören" (Konrád 89).

Peter Handke, der berühmte österreichische Schriftsteller, dessen Mutter Slowenin war und der aus Interesse an seinen Wurzeln Slowenien viel bereist und mit Sympathie darüber geschrieben hatte, war bei der Mitteleuropa-Idee ganz anderer Meinung. Nach dem Fall der Berliner Mauer habe der Wunsch nach Mitteleuropa die Slowenen dazu gebracht, sich von Jugoslawien abzuspalten, und diesen Schritt lehnte Handke polemisch und vehement ab: "Nein, das zunehmende Wegdriften so vieler Slowenen von ihrem großen Jugoslawien, 'hin zu Mitteleuropa', oder 'zu Europa', oder 'zum Westen', nahm ich lange nur als bloße Laune [...] Denn nichts, gar nichts, drängte bis dahin in der Geschichte des slowenischen Lands zu einem Staat-Werden. Nie, niemals hatte das slowenische Volk so etwas wie einen Staatentraum" (Handke 38-39). Seine sarkastische Aussage über Mitteleuropa als meteorologischer Begriff fiel in einem Interview für die Toriner Tageszeitung La Stampa am 6. Januar 1987 und dann noch im gleichen Jahr in Slowenien, auf einer Pressekonferenz in Vilenica, wo ihm ein Preis verliehen wurde. Dieser Auftritt wirbelte viel Staub auf, schließlich wird der Vilenica-Preis für schriftstellerische Leistungen vergeben, die im Geiste Mitteleuropas entstehen. Die Aussage ist nicht autorisiert, denn sie taucht in journalistischen Texten auf, doch beginnt sie immer mit dem Satz "Mitteleuropa hat für mich lediglich eine meteorologische Bedeutung", und sie endet mit: "Mitteleuropa, ein Begriff, den ich nie in einer ideologischen Bedeutung verwenden würde, ist eine Angelegenheit, die mit meteorologischen Erscheinungen verbunden ist“.

Sobald wir 'Meteorologie' durch den recht verwandten Begriff 'Geographie' ersetzen, stellen wir fest, dass Handke direkt mit Kundera polemisiert. Dieser weist nämlich ausdrücklich darauf hin, dass die Mitteleuropäer den Ausdruck 'Europa' auf besondere Weise verstehen: "Für sie ist Europa kein geographischer Begriff, sondern ein geistiger, gleichbedeutend mit dem Wort 'Westen' "(Kundera 133). Handke dagegen beharrt im mittleren Teil seiner Aussage spöttisch auf Geographie, denn er spricht über seine Wanderungen in den Julischen Alpen, von den Wolken über ihnen, über die Landschaften nördlich der Alpen, wo es regnerisch und bewölkt ist, und über den slowenischen Karst im Süden, wo der Wind weht, die Sonne scheint und Kiefern und Feigen wachsen. Absichtlich verzichtet er auf die metaphysische Ladung des Ausdrucks und beharrt auf dem rein Konkreten. Es scheint, als sei ihm als ausgewiesenem Individualisten oder sogar Subjektivisten jeder Auftritt in der Gruppe fremd, jede Gruppeneuphorie, und auch, dass ihm schon die jugoslawische Föderation die verwirklichte Idee des Zusammenlebens verschiedener Völker bedeutete und er sich mit ihrem Zerfall nicht versöhnen konnte:

Slowenien gehörte für mich seit je zu dem großen Jugoslawien, das südlich der Karawanken begann und weit unten, zum Beispiel am Ohridsee bei den byzantinischen Kirchen und islamischen Moscheen vor Albanien oder in den makedonischen Ebenen vor Griechenland, endete (Handke 19-20). 
MUZIKOLOŠKI ZBORNIK • MUSICOLOGICAL ANNUAL XL

Auf Handkes meteorologische Provokationen antwortete Drago Jančar. Er räumte ein, dass die mitteleuropäische Identität manchmal etwas zwanghaft gesucht wurde und dass es vielleicht schon zu viel des allseitigen Geredes über das Thema gebe, vor allem, weil nicht nur die Qualifizierten sich zu Wort meldeten: "In den Mund nahmen es auch viele professionelle Phrasendrescher und zerkauten es zu einer formlos-abstrakten, rhetorischen Masse" (Jančar 89). Doch das Wesen der MitteleuropaIdee hatte für ihn noch immer sein Gewicht erhalten. Noch einmal machte er auf das Phänomen des 'Kulturbabylons' aufmerksam, d. h. der Vielfalt, des Gesinnungspluralismus, der Zersplitterung und der Vielsprachigkeit. Für ihn sind das Werte, die trotz der gegenläufigen und gewaltsamen Macht der Geschichte, die über diese Gegenden zieht, Hoffnung geben, "neue Hoffnung “. Nach dem Fall der Berliner Mauer und der slowenischen Verselbständigung versah Jančar diesen Artikel mit einem Zusatz, in dem er feststellt, dass sich die Utopie verwirklicht habe, es aber nicht gesagt sei, dass die Slowenen in dieser neuen Zeit bestehen können, da sie die lange Sklaverei geistig verkrüppelt haben mag. Im Nachhinein sei das Thema Mitteleuropa nur noch das Zeugnis einer ideologischen Zeit. "Was uns wirklich verbindet im mitteleuropäischen Raum, ist ziemlich wage. Heute zeigt sich plötzlich, dass uns mehr der Widerstand gegen seine Teilung verband, als verwandte kulturelle Fragen " Jančar 93). Das ist ein ziemlich pessimistischer Gedanke, der aber bestätigt, dass die publizistische Behandlung des Themas Mitteleuropa nun zu Ende ist.

Die publizistische Verwendung des Begriffs flackerte auf und verebbte wieder; nach dem Fall der Berliner Mauer und in der Zeit der Annäherung an die Europäische Union bestand kein Bedarf mehr danach. Und doch war diese Denkart über Mitteleuropa, die 1984 in der Zeitschrift Nova revija mit der Übersetzung von Kunderas Artikel Die Tragödie Mitteleuropas begann, von allen anderen in Slowenien die intensivste und trug den höchsten Grad an Bewusstsein in sich. Im Rahmen der vorliegenden Studie schien es notwendig, einige dieser Artikel mit der gleichen Aufmerksamkeit zu behandeln, als ginge es um literarische Texte. Das Argument für diesen Ansatz war, dass diese publizistischen Texte (Essays) schließlich von Literaten verfasst wurden und dass mindestens ein Teil dieses Materials auch in ihre Literatur mit einfloss. Wäre diese Studie nach einer strengeren Systematik verfahren, dann hätte sie nur literarische und wissenschaftliche Texte berücksichtigt, doch dann wäre ein wesentlicher Teil der Entwicklung des Begriffs 'Mitteleuropa' einfach unberücksichtigt geblieben.

Wir haben drei Verwendungen des Begriffs 'Mitteleuropa' behandelt - die wissenschaftliche, literarische und publizistische - und auf den ersten Blick lebt jede ihr eigenes Leben. Doch nur auf den ersten Blick, denn es gibt Wechselwirkungen zwischen ihnen. Die literaturwissenschaftliche Verwendung ist aufgrund der schwachen Textbasis zurückhaltend und von niedriger Frequenz, sie lebt erst auf beim literarischen Expressionismus, der sich als eminente literarische Richtung Mitteleuropas erweist. Die literarische Verwendung taucht evident gerade dort auf, im Expressionismus, doch hat sie kein Bewusstsein von sich: Die expressionistischen Schriftstel- 
ler waren sich nicht bewusst, dass sie mitteleuropäische Themen behandelten, einige zählten sich nicht einmal zum Expressionismus (z. B. Slavko Grum). Die publizistische Verwendung entstand formal zwar als aktualistische, ideologische Antwort auf die unerträgliche Herrschaft der kommunistischen Regimes, die von der Sowjetunion nach der Besetzung Osteuropas Ende des 2. Weltkriegs errichtet wurden, doch berief sie sich gleichzeitig unvermeidlich auch auf frühere Feststellungen der wissenschaftlichen und literarischen Verwendung.

In den kommenden Jahren wird sich vor allem die Wissenschaft mit diesem Gebiet befassen. Sie muss eine grundlegende Systematik schaffen, bei der sich sofort mindestens zwei miteinander verbundene, große Fragen stellen: 1) Kann nach dem 2. Weltkrieg überhaupt noch mitteleuropäische Literatur geschrieben werden und 2) kann mitteleuropäische Literatur nur im Spannungsfeld zum habsburgischen Mythos entstehen? Anders ausgedrückt: Schreibt Drago Jančar mitteleuropäische Literatur, da sich seine Helden in einer historischen und geographischen Welt bewegen, die genau so ist? Oder geht es nur noch um eine postmoderne Reflexion, da Jančar nicht mehr wesentlich, existentiell an den habsburgischen Mythos gebunden ist, so wie auch kein anderer Schriftsteller nach dem 2. Weltkrieg?

\section{Literatur}

"Budapest Roundtable, The." Cross Currents 10 (1991): 17-30.

Busek, Erhard und Gerhard Wilfinger (Hrsg.). Aufbruch nach Mitteleuropa. Rekonstruktion eines versunkenen Kontinents. Wien: Edition Atelier, 1987.

Cornis-Pope, Marcel und John Neubauer. Towards a History of the Literary Cultures in East-Central Europe. Theoretical Reflections. New York: American Council of Learned Societies Occasional Paper, 2002.

Grafenauer, Ivan. Zgodovina novejšega slovenskega slovstva.1. Od Pohlina do Prešerna. Ljubljana: Katoliška bukvarna, 1909.

Handke, Peter. Abschied des Träumers vom Neunten Land. Frankfurt: Suhrkamp, 1991.

Jančar, Drago. "Srednja Evropa med meteorologijo in utopijo." Srednja Evropa. Peter Vodopivec (Hrsg.), o. c. 87-94.

Konrád, György. "Der Traum von Mitteleuropa." Aufbruch nach Mitteleuropa. Erhard Busek und Gerhard Wilfinger (Hrsg.), o. c. 87-97.

Konstantinović, Zoran (Hrsg.). 'Expressionismus' im europäischen Zwischenfeld. Innsbruck: Innsbrucker Gesellschaft zur Pflege der Geisteswissenschaften, 1978.

Konstantinović, Zoran. "Expressionismus in Südosteuropa. Fragen der Gemeinsamkeiten." Actes du VIII Congrès de l'Association Internationale de Littérature Comparée. 1. Stuttgart: Erich Bieber, 1980. 715-719.

Kos, Janko. "Srednja Evropa kot literarnozgodovinski problem."Srednja Evropa. Peter Vodopivec (Hrsg.), o. c. 41-53.

Kralj, Lado. Ekspresionizem. Ljubljana: DZS, 1986.

Kundera, Milan. "Die Tragödie Mitteleuropas." Aufbruch nach Mitteleuropa. Erhard Busek und Gerhard Wilfinger (Hrsg.), o. c. 133-144. 
MUZIKOLOŠKI ZBORNIK • MUSICOLOGICAL ANNUAL XL

Magris, Claudio. Il mito asburgico nella letteratura austriaca moderna. Milano: Einaudi, 1963.

Magris, Claudio. Der habsburgische Mythos in der österreichischen Literatur. Wien: Zsolnay Verlag, 2000.

Le Rider, Jacques. Mitteleuropa. Auf den Spuren eines Begriffs. Wien: Deuticke, 1994. Naumann, Friedrich. Mitteleuropa. Berlin: Reimer, 1915.

Petrè, Fran. "Slovenska književnost med obema vojnama." Nova obzorja. 7.11 (1954): 633-647.

Vodopivec, Peter (Hrsg.). Srednja Evropa. Ljubljana: Mladinska knjiga, 1991. 